\title{
Avascular Placental Villi
}

National Cancer Institute

\section{Source}

National Cancer Institute. Avascular Placental Villi. NCI Thesaurus. Code C118152.

Focus of two or more placental terminal villi showing a total loss of villous capillaries and bland hyaline fibrosis of the villous stroma. Stromal karyorrhexis may or may not be evident. 